\title{
Comparison of mammography and ultrasound in detecting residual disease following bioptic lumpectomy in breast cancer patients
}

\author{
XIUFENG WU ${ }^{1 *}$, QINGZHONG LIN ${ }^{1 *}$, JIANPING LU $^{3 *}$, GANG CHEN $^{3}$, YI ZENG $^{1}$, \\ YINGLAN LIN $^{1}$, YING CHEN ${ }^{4}$, YAOQIN WANG ${ }^{5}$ and JUN YAN $^{2}$ \\ ${ }^{1}$ Department of Surgery, Fujian Provincial Tumor Hospital, Teaching Hospital of Fujian Medical University, Fuzhou, \\ Fujian 350014; ${ }^{2}$ Department of General Surgery, Nanfang Hospital, Southern Medical University, Guangzhou, \\ Guangdong 510515; Departments of ${ }^{3}$ Pathology, ${ }^{4}$ Radiology and ${ }^{5}$ Ultrasound, Fujian Provincial Tumor Hospital, \\ Teaching Hospital of Fujian Medical University, Fuzhou, Fujian 350014, P.R. China
}

Received July 13, 2015; Accepted November 6, 2015

DOI: $10.3892 / \mathrm{mco} .2016 .729$

\begin{abstract}
Surgical biopsy is a method for diagnosing breast cancer. The aim of this study was to prospectively evaluate the relative accuracies of mammography (MMG) and ultrasound (US) in predicting residual disease following bioptic lumpectomy. Each prediction method was compared with the gold standard of surgical pathology. The results of MMG and US from 312 consecutive breast cancer patients diagnosed by surgical excision were analyzed. All the patients underwent re-excision mastectomy or lumpectomy and the imaging results were compared with the histopathological findings. The accuracy and sensitivity of each modality were investigated. A total of 312 patients with 312 primary breast cancers were investigated. Residual disease was identified in 118 patients. Of the 118 cases with residual disease, MMG and US were able to detect $77(65.3 \%)$ and 32 (27.1\%), respectively (Chi-square $\mathrm{P}<0.001)$. MMG was also more sensitive compared with US in estimating residual ductal carcinoma in situ (DCIS) (94.2 vs. 33.3\%, respectively; $\mathrm{P}<0.001)$. MMG was more accurate compared with US in detecting residual disease following bioptic lumpectomy and the diagnostic accuracy of MMG was associated with the presence of residual DCIS.
\end{abstract}

\section{Introduction}

Although the majority of breast cancers are currently diagnosed by needle biopsy, there are valid exceptions for which this may

Correspondence to: Dr Xiufeng Wu, Department of Surgery, Fujian Provincial Tumor Hospital, Teaching Hospital of Fujian Medical University, Fuzhou, Fujian 350014, P.R. China

E-mail:wxf200104@hotmail.com

${ }^{*}$ Contributed equally

Key words: mammography, ultrasound, residual disease, lumpectomy, breast cancer not be possible or necessary, and thus require surgical excision (1). In addition, surgical excision is the first biopsy choice for breast cancer in certain centers, due to cultural reasons. Approximately half of breast cancer patients in China are diagnosed by excision biopsy (2). Following diagnosis, a proportion of patients opt directly for surgical excision. Surgical biopsy of suspicious breast lesions enables histological diagnosis and facilitates appropriate treatment planning. However, the use of excision biopsy raises several issues $(3,4)$, among which is the residual disease that may be associated with a higher incidence of recurrence. Particularly for patients who are candidates for breast conservation, the presence of residual disease may require multiple re-excisions (5).

The incidence of residual disease following initial excisional biopsy of breast cancer is variable, ranging from 45 to $70 \%$ in the literature $(6,7)$. Accurate assessment of residual tumor following bioptic lumpectomy is crucial for subsequent treatment planning. Patients with no residual disease following bioptic lumpectomy may only require excising and pathologically evaluating the cavity margins instead of aggressive re-excision mastectomy.

Mammography (MMG) and ultrasound (US) are currently the most commonly used imaging modalities for the diagnosis of primary breast cancer, with a fairly high accuracy (8-13). However, it may be more difficult to detect residual disease following lumpectomy with these modalities. The most significant concerns are associated with the fact that the process of wound healing following surgical excision may lead to changes in breast architecture (14), which may affect the diagnostic accuracy of the imaging modalities (15) and the identification of residual tumor. In the present study, we aimed to evaluate the accuracy of MMG and breast US in detecting residual tumor following bioptic lumpectomy in breast cancer patients.

\section{Patients and methods}

Ethics statement. Patients with primary breast cancer who were initially diagnosed by surgical excision followed by re-excision breast-conserving surgery or mastectomy were recruited to participate in this study, which was approved by 
the Institutional Review Board of Fujian Provincial Tumor Hospital. Written informed consent was obtained from all the patients prior to their participation in the present study.

Study population. All the primary breast cancer cases from the surgical pathology files of the Fujian Provincial Tumor Hospital over a 10-year period (2003-2012) were reviewed. The study population came from two different sources: A proportion of the patients were diagnosed at our center, whereas others were referred to our institution for definitive surgery after undergoing excision biopsy performed at an external institution. All the patients had primary breast cancer diagnosed by excision biopsy.

Diagnostic imaging equipment. MMG was performed using two digital full-field instruments, namely Senographe 2000D (GE Healthcare, Munich, Germany) and Selenia (Hologic GmbH, Frankfurt am Main, Germany).

Sonography was performed using a linear transducer with a 50-mm width and a frequency of $12 \mathrm{MHz}$, using Philips models iU22 and HD11 (Philips GmbH Healthcare, Hamburg, Germany). Real-time spatial compound imaging (CT) in combination with adaptive image processing (XRES) was a method to analyze sonographic criteria for the differentiation of benign and malignant breast lesions.

MMG and breast US were performed to evaluate residual tumor prior to reoperation (mastectomy or breast-conserving surgery) as part of routine patient care at the discretion of the breast surgeon. Magnetic resonance imaging (MRI) is not routinely used as part of patient care at our center; therefore, it was not included in this study. MMG evaluation included standard craniocaudal and mediolateral oblique views. All the mammograms were reviewed independently by two radiologists experienced in the interpretation of breast MMG based on the morphological pattern and density on the MMG images. Similarly, another two radiologists experienced in the interpretation of breast US, independently reviewed all US images in terms of echoic structures and acoustic shadowing. The radiologists were blinded to any clinical or histopathological information of the patients. Following diagnostic imaging, all the patients underwent reoperation (mastectomy or breast-conserving surgery) (Fig. 1). The re-excision specimens were sent to the histopathology laboratory for histological examination by two histopathologists specialized in breast pathology. Residual disease was defined as microscopically confirmed invasive or in situ carcinoma identified within the mastectomy or re-excision specimens.

Statistical analysis. The diagnostic performance of MMG and breast US was evaluated by calculating the accuracy, sensitivity and negative predictive values. Categorical variables were compared between the two groups using the Chi-square test. IBM SPSS software, version 20 (IBM Corp, Armonk, NY, USA) was used for the statistical analyses. P-values $<0.05$ were considered to indicate statistically significant differences.

\section{Results}

Patient characteristics. A total of 320 patients were considered as eligible for this study, 8 of whom did not participate

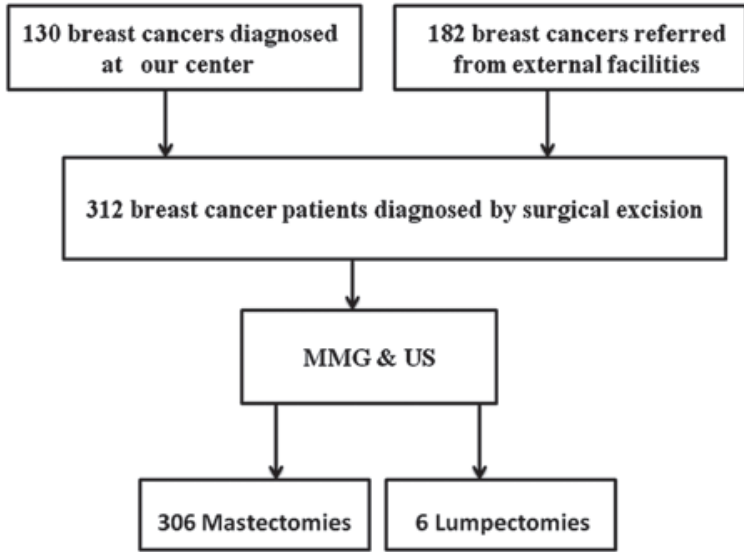

Figure 1. Treatment course of 312 breast cancer patients who required re-excisions or mastectomy following initial surgical excision. MMG, mammography; US, ultrasound.

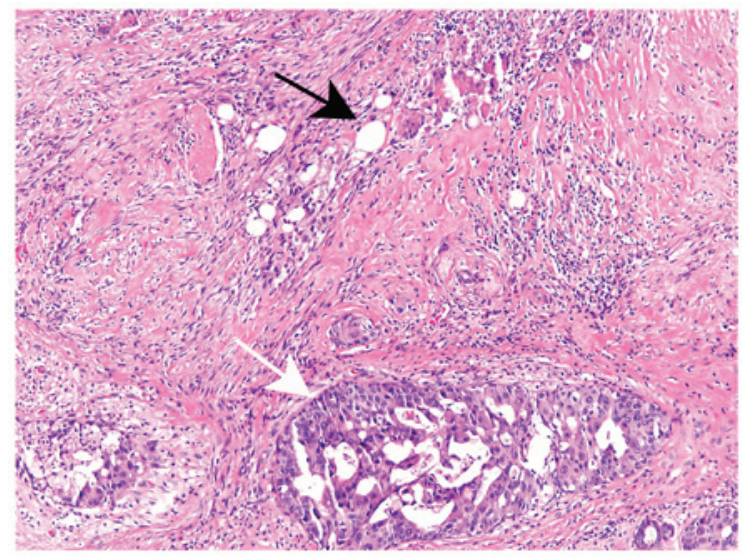

Figure 2. A 56-year-old breast cancer patient with residual disease of the right breast following lumpectomy. Histological section showing the incision site (black arrow) and a focus of residual ductal carcinoma in situ in an adjacent location (white arrow) (haematoxylin and eosin staining; magnification, x10).

due to scheduling constraints. Of the 312 eligible patients, $130(41.7 \%)$ were diagnosed in our center and were clinically and radiologically considered to have benign disease; however, the subsequent surgical excision and histological examination revealed a positive result. The remaining 182 patients $(58.3 \%)$ were referred from other institutions following diagnosis; radiological information was not available for these patients. The median patient age was 49 years (range, 27-85 years). All the patients underwent MMG and breast US prior to reoperation. The re-excision procedure was mastectomy in 306 cases and lumpectomy in the remaining 6 cases, based on the surgeon's discretion and the patient's preference, irrespective of imaging findings. Due to inherent cultural barriers and cancer fatalism in Chinese women, the majority of the patients opted for mastectomy upon breast cancer diagnosis.

Residual disease was confirmed in 118 of 306 patients by final pathology in re-excision mastectomy specimens. There was no residual disease in the 6 re-excision lumpectomy specimens. Residual invasive ductal carcinoma (IDC) or ductal carcinoma in situ (DCIS) alone were found in 15.2 and $58.5 \%$ of the cases, respectively, whereas $17.8 \%$ of the patients were 
Table I. Final pathological diagnosis in cases with residual disease on re-excision specimens.

\begin{tabular}{|c|c|c|c|c|}
\hline Residual disease cases & Mixed IDC+DCIS & DCIS & IDC & Others $^{\mathrm{a}}$ \\
\hline 118 & $21(17.8 \%)$ & $69(58.5 \%)$ & $18(15.2 \%)$ & $10(8.5 \%)$ \\
\hline
\end{tabular}

${ }^{a}$ Residual invasive lobular carcinoma and mucinous carcinoma. IDC, invasive ductal carcinoma; DCIS, ductal carcinoma in situ.

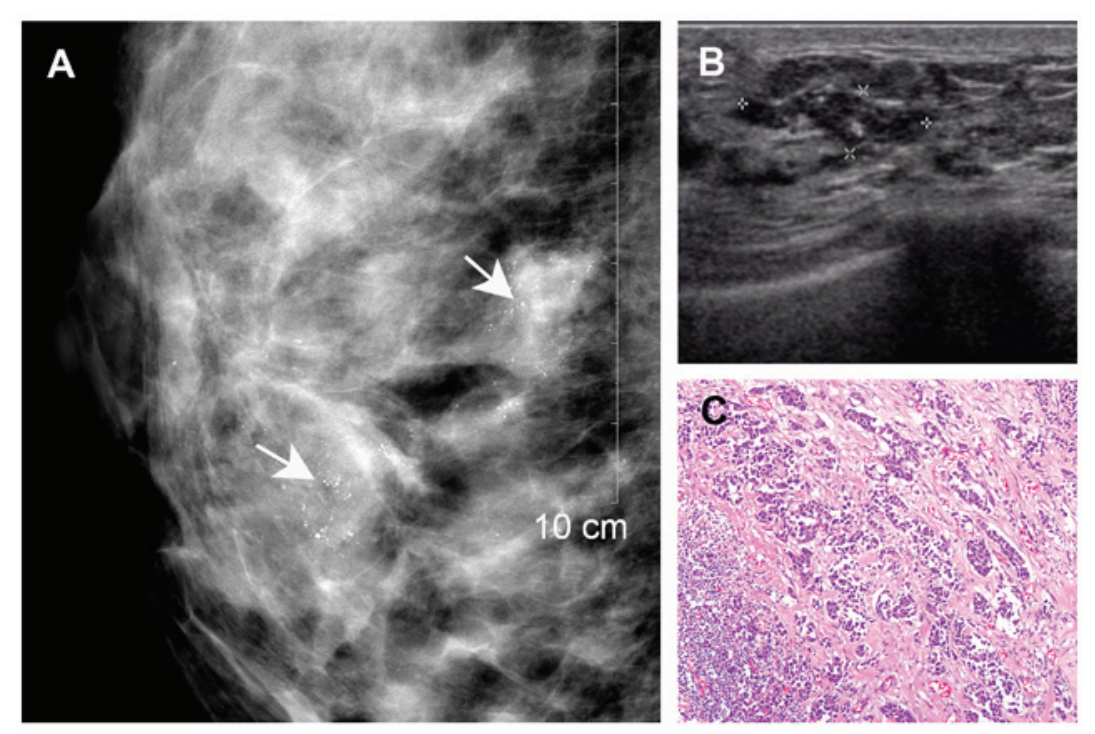

Figure 3. A 42-year-old breast cancer patient with residual disease of the right breast following lumpectomy. (A) Mammography revealed dense breast tissue with multiple clustered microcalcifications (arrows). (B) Ultrasonography revealed an indistinct hypoechoic area with internal hyperechoic foci (asterisks), corresponding to the mammographically visualized microcalcifications. (C) Histological diagnosis of residual invasive ductal carcinoma (haematoxylin and eosin staining; magnification, $\mathrm{x} 10$ ).
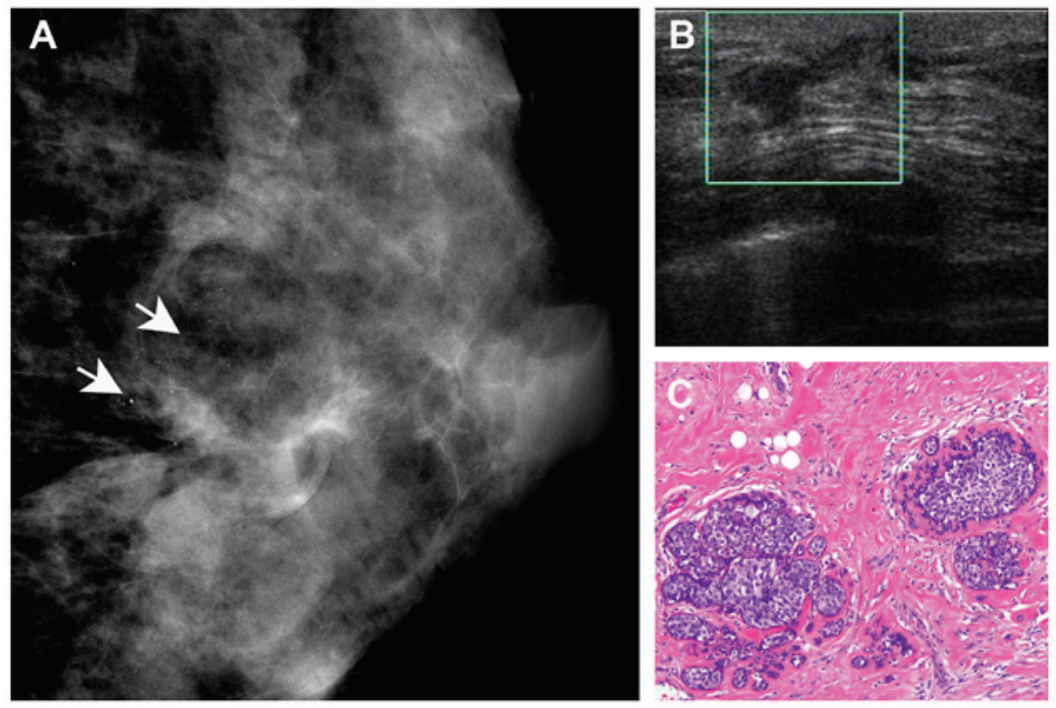

Figure 4. A 35-year-old breast cancer patient with residual disease of the left breast following lumpectomy. (A) Mammography revealed heterogeneously dense breast tissue with scattered microcalcifications (arrows). (B) Ultrasonography revealed irregular tissue distribution in the left breast (square). (C) Histological diagnosis of residual ductal carcinoma in situ (haematoxylin and eosin staining; magnification, x10).

allocated to the IDC+DCIS group. Other types of tumors in this study included residual invasive lobular carcinoma and mucinous carcinoma, occurred in $8.5 \%$ of the cases (Table I). Residual disease was not identified in 194 of the 312 cases, despite extensive sectioning of the re-excision specimens (16).
Residual tumor was usually present in adjacent tissues, rather than within the excision cavity (Fig. 2).

Identifying residual disease with imaging modalities. Both MMG and breast US detected residual disease in 20 of the 
Table II. Diagnostic accuracy of MMG in detecting residual disease.

\begin{tabular}{|c|c|c|c|c|c|c|c|c|}
\hline \multirow[b]{2}{*}{ MMG results } & \multicolumn{2}{|c|}{ Residual disease } & \multirow[b]{2}{*}{ Total } & \multirow[b]{2}{*}{ Sensitivity } & \multirow[b]{2}{*}{ Specificity } & \multirow[b]{2}{*}{ PPV } & \multirow[b]{2}{*}{ NPV } & \multirow[b]{2}{*}{ Accuracy } \\
\hline & Present & Absent & & & & & & \\
\hline Present & 77 & 9 & 86 & $65.3 \%$ & $95.4 \%$ & $89.5 \%$ & $81.9 \%$ & $83.9 \%$ \\
\hline Absent & 41 & 185 & 226 & (77/118) & (185/194) & $(77 / 86)$ & $(185 / 226)$ & $(262 / 312)$ \\
\hline Total & 118 & 194 & 312 & & & & & \\
\hline
\end{tabular}

MMG, mammography; PPV, positive predictive value; NPV, negative predictive value.

Table III. Diagnostic accuracy of US in detecting residual disease.

\begin{tabular}{lccccccccc}
\hline & \multicolumn{2}{c}{ Residual disease } & & & & & \\
\cline { 2 - 6 } US results & Present & Absent & & Total & Sensitivity & Specificity & PPV & NPV & Accuracy \\
\hline Present & 32 & 3 & 35 & $27.1 \%$ & $98.5 \%$ & $91.4 \%$ & $68.9 \%$ & $71.5 \%$ \\
Absent & 86 & 191 & 277 & $(32 / 118)$ & $(191 / 194)$ & $(32 / 35)$ & $(191 / 277)$ & $(223 / 312)$ \\
Total & 118 & 194 & 312 & & & & & \\
\hline
\end{tabular}

US, ultrasound; PPV, positive predictive value; NPV, negative predictive value.

118 cases that were were correlated with the pathological findings (Fig. 3). However, 28 cases with pathologically confirmed residual disease were not detected by either MMG or US. Of the 86 cases with residual disease identified by MMG, 77 were correlated with histopathological findings. The remaining 9 cases did not have residual disease on final pathology. US detected residual disease in 35 cases, of which 32 were confirmed by pathology, whereas the remaining 3 cases turned out to be negative for residual disease on final pathology. A total of 57 cases with residual disease identified on MMG were not detected by US (Fig. 4). However, 12 cases of residual disease detected by US were not identified on MMG. As regards the 89 cases with residual DCIS, 65 were accurately detected by MMG, whereas only 23 were accurately detected by US.

Sensitivity and accuracy of MMG and US. We next evaluated the sensitivity and accuracy of the two methods for the detection of residual disease in re-excision specimens. The overall performance of each of the imaging modalities is summarized in Table IV.MMG was more sensitive, detecting 77/118 (65.3\%) of all residual disease cases. This was significantly superior to US, which detected 32/118 (27.1\%) residual disease cases $(\mathrm{P}<0.001)$ (Tables II-IV). As regards the 89 cases with residual DCIS, the sensitivity of MMG was also significantly superior to that of US [65/69 (94.2\%) vs. 23/69 (33.3\%) cases of residual disease detected, respectively] (Table V). These data clearly demonstrate that MMG is a reliable method for identifying residual disease, particularly DCIS.

\section{Discussion}

Surgical biopsy is widely used in China, due to inherent cultural barriers and cancer fatalism in Chinese women. Accurate
Table IV. Results of data synthesis.

\begin{tabular}{lccc}
\hline Characteristics & MMG $(\%)$ & US (\%) & P-value $^{\mathrm{a}}$ \\
\hline Accuracy & 83.9 & 71.5 & $<0.001$ \\
Sensitivity & 65.3 & 27.1 & $<0.001$ \\
Specificity & 95.4 & 98.5 & 0.07 \\
NPV & 81.9 & 68.9 & 0.001 \\
PPV & 89.5 & 91.4 & 0.5 \\
\hline
\end{tabular}

${ }^{a}$ Chi-square. MMG, mammography; US, ultrasound; PPV, positive predictive value; NPV, negative predictive value.

Table V. Sensitivity and accuracy of MMG and US in the detection of residual ductal carcinoma in situ.

\begin{tabular}{llll}
\hline Variables & \multicolumn{1}{c}{ MMG (\%) } & \multicolumn{1}{c}{ US (\%) } & P-value $^{\mathrm{a}}$ \\
\hline Sensitivity & $94.2(65 / 69)$ & $33.3(23 / 69)$ & $<0.001$ \\
Accuracy & $95.8(113 / 118)$ & $58.5(69 / 118)$ & $<0.001$ \\
\hline
\end{tabular}

${ }^{a}$ Chi-square. MMG, mammography; US, ultrasound.

prediction of residual disease following surgical excision is crucial for treatment selection. The present study compared MMG and US in detecting residual disease following bioptic lumpectomy in Chinese women. Our results demonstrated that the sensitivity, accuracy and negative predictive value were significantly higher with MMG compared with US. Further 
analyses suggested that sensitivity and accuracy were also higher with MMG regarding the detection of residual DCIS following bioptic lumpectomy. Therefore, our data suggest that MMG was more accurate compared with breast US in identifying residual tumor following bioptic lumpectomy.

Currently, MMG and US are the most commonly used imaging modalities for primary breast cancer diagnosis, although it remains unclear which modality is superior for accurate diagnosis of breast abnormalities. Berg et al (8), Bosch et al (9) and Madjar et al (12) confirmed the sonographic superiority to MMG in detecting primary tumors. However, studies comparatively analyzing the diagnostic accuracy of MMG and sonography demonstrated that the two modalities perform equally well in detecting primary breast cancer $(11,17)$, or that MMG is superior to breast US in the accurate diagnosis of primary breast cancer $(2,18)$. Although it has been reported that US was more accurate compared with MMG in predicting residual tumor size following neoadjuvant chemotherapy (19), there is no available literature regarding the optimal method for accurate assessment of residual disease following bioptic lumpectomy. Identifying residual tumor following lumpectomy may be even more challenging. In this prospective study, we observed that a greater proportion of residual tumors following bioptic lumpectomy were detected using MMG rather than US, whereas MMG was more accurate compared with breast US in identifying residual tumor following bioptic lumpectomy.

The presence of DCIS was a significant predictor of an increased likelihood of residual disease at re-excision following breast-conserving therapy (20-25). In our series, residual DCIS accounted for $58.5 \%(69 / 118)$ of the cases, indicating that DCIS is also a risk factor for residual disease following bioptic lumpectomy. Of the 69 residual DCISs, 65 (94.2\%) were visible on MMG as abnormal lesions, with or without microcalcifications. Compared with MMG, only 23 residual disease cases with DCIS were detected by US, which indicates that MMG may be more effective compared with US in the detection of residual DCIS $(26,27)$.

In this study, $23.7 \%$ of the residual tumors could not be detected by either MMG or US. Since MRI has been proven to have a better sensitivity for evaluating the extent of breast cancer and detecting additional breast lesions compared with conventional visualizing methods (28), MRI is very useful for evaluating residual disease that cannot be detected using US or MMG. Further investigations should focus on evaluating the accuracy of MRI in detecting residual disease following bioptic lumpectomy.

The superiority of $\mathrm{MMG}$ regarding residual disease detection following bioptic lumpectomy has not been documented. MMG in our study cohort was associated with a high detection rate of residual disease following bioptic lumpectomy. Thus, the use of MMG-guided stereotactic biopsy may enable complete removal of malignant foci with clear excision margins, resulting in a reduced rate of second operations. Moreover, the use of preoperative MMG may enable accurate evaluation of residual disease size following bioptic lumpectomy, allowing for selecting eligible candidates for breast-conserving surgery. Finally, our results further confirmed a previous study reporting that MMG is useful in early detection of breast cancer (29).
The present study had certain limitations. First, we only enrolled breast cancer patients diagnosed by initial surgical excision followed by mastectomy or lumpectomy, without considering the time interval between surgical excision and re-excision; however, the incidence of residual disease in breast cancer may be affected by the time interval between lumpectomy and subsequent re-excision (14). Second, a proportion of the patients were referred from other facilities, and information on primary tumors, such as palpability, size, localization and biology, were not available; however, tumor size and biology are significant predictive factors for residual disease (30).

Our prospective analysis of the ability of MMG and breast US to identify residual disease following bioptic lumpectomy, compared with surgical pathology evaluation of the residual tumor, demonstrated that $65.3 \%$ of residual tumors were accurately detected using MMG, compared with $27.1 \%$ using breast US. The diagnostic accuracy of MMG was associated with the presence of residual DCIS. Our results underscore the significance of MMG in treatment selection and residual disease localization following bioptic lumpectomy.

\section{Acknowledgements}

The present study was supported by grants from the National Natural Science Foundation of China (no. 81272574), the Natural Science Foundation of Fujian Province (nos. 2014J01300 and 2014J05086), the Medical Innovation Program of Fujian Province (no. 2012-CXB-7), the Program from Education Bureau of Fujian Province (no. JB13127), and the Scientific Research Foundation for High Level Talents in Nanfang Hospital of Southern Medical University.

\section{References}

1. Corben AD, Edelweiss M and Brogi E: Challenges in the interpretation of breast core biopsies. Breast J 16 (Suppl 1): S5-S9, 2010.

2. Fan L, Strasser-Weippl K, Li JJ, St Louis J, Finkelstein DM, Yu KD, Chen WQ, Shao ZM and Goss PE: Breast cancer in China. Lancet Oncol 15: e279-e289, 2014.

3. Osanai T, Gomi N, Wakita T, Yamashita T, Ichikawa W, Nihei Z and Sugihara K: US-guided core needle biopsy for breast cancer: Preliminary report. Jpn J Clin Oncol 30: 65-67, 2000.

4. Fishman JE, Milikowski C, Ramsinghani R, Velasquez MV and Aviram G: US-guided core-needle biopsy of the breast: How many specimens are necessary? Radiology 226: 779-782, 2003.

5. Menes TS, Tartter PI, Bleiweiss I, Godbold JH, Estabrook A and Smith SR: The consequence of multiple re-excisions to obtain clear lumpectomy margins in breast cancer patients. Ann Surg Oncol 12: 881-885, 2005.

6. Jardines L, Fowble B, Schultz D, Mackie J, Buzby G, Torosian M, Daly J, Weiss M, Orel S and Rosato E: Factors associated with a positive reexcision after excisional biopsy for invasive breast cancer. Surgery 118: 803-809, 1995.

7. Gwin JL, Eisenberg BL, Hoffman JP, Ottery FD, Boraas M and Solin LJ: Incidence of gross and microscopic carcinoma in specimens from patients with breast cancer after re-excision lumpectomy. Ann Surg 218: 729-734, 1993.

8. Berg WA, Gutierrez L, NessAiver MS, Carter WB, Bhargavan M, Lewis RS and Ioffe OB: Diagnostic accuracy of mammography, clinical examination, US and MR imaging in preoperative assessment of breast cancer. Radiology 233: 830-849, 2004.

9. Bosch AM, Kessels AG, Beets GL, Rupa JD, Koster D, van Engelshoven JM and von Meyenfeldt MF: Preoperative estimation of the pathological breast tumour size by physical examination, mammography and US: A prospective study on 105 invasive tumours. Eur J Radiol 48: 285-292, 2003. 
10. Hieken TJ, Harrison J, Herreros J and Velasco JM: Correlating sonography, mammography and pathology in the assessment of breast cancer size. Am J Surg 182: 351-354, 2001.

11. Kald BA, Boiesen P, Ronnow K, Jonsson PE and Bisgaard T: Preoperative assessment of small tumours in women with breast cancer. Scand J Surg 94: 15-20, 2005.

12. Madjar H, Ladner HA, Sauerbrei W, Oberstein A, Prömpeler H and Pfleiderer A: Preoperative staging of breast cancer by palpation, mammography and high-resolution US. US Obstet Gynecol 3: 185-190, 1993.

13. Yang WT, Lam WW, Cheung H, Suen M, King WW and Metreweli C: Sonographic, magnetic resonance imaging and mammographic assessments of preoperative size of breast cancer. J US Med 16: 791-797, 1997.

14. Wiley EL, Diaz LK, Badve S and Morrow M: Effect of time interval on residual disease in breast cancer. Am J Surg Pathol 27: 194-198, 2003

15. Wiratkapun C, Wibulpholprasert B, Wongwaisayawan S and Pulpinyo K: Nondiagnostic core needle biopsy of the breast under imaging guidance: Result of rebiopsy. J Med Assoc Thai 88: 350-357, 2005.

16. Wiley E and Keh P: Diagnostic discrepancies in breast specimens subjected to gross reexamination. Am J Surg Pathol 23: 876-879, 1999.

17. Wang JT, Chang LM, Song X, Zhao LX, Li JT, Zhang WG Ji YB, Cai LN, Di W and Yang XY: Comparison of primary breast cancer size by mammography and sonography. Asian Pac J Cancer Prev 15: 9759-9761, 2014.

18. Golshan M, Fung BB, Wiley E, Wolfman J, Rademaker A and Morrow M: Prediction of breast cancer size by US, mammography and core biopsy. Breast 13: 265-271, 2004.

19. Keune JD, Jeffe DB, Schootman M, Hoffman A, Gillanders WE and Aft RL: Accuracy of ultrasonography and mammography in predicting pathologic response after neoadjuvant chemotherapy for breast cancer. Am J Surg 199: 477-484, 2010

20. Boyages J, Recht A, Connolly J, Schnitt SJ, Gelman R, Kooy H, Love S, Osteen RT, Cady B, Silver B, et al: Early breast cancer: Predictors of breast recurrence for patients treated with conservative surgery and radiation therapy. Radiother Oncol 19: 29-41, 1990.

21. Chan KC, Knox WF, Sinha G, Gandhi A, Barr L, Baildam AD and Bundred NJ: Extent of excision margin width required in breast conserving surgery for ductal carcinoma in situ. Cancer 91: $9-16,2001$.
22. Holland R, Connolly JL, Gelman R, Mravunac M, Hendriks JH, Verbeek AL, Schnitt SJ, Silver B, Boyages J and Harris JR: The presence of an extensive intraductal component following a limited excision correlates with prominent residual disease in the remainder of the breast. J Clin Oncol 8: 113-118, 1990.

23. Aziz D, Rawlinson E, Narod SA, Sun P,Lickley HL, McCready DR and Holloway CM: The role of reexcision for positive margins in optimizing local disease control after breast-conserving surgery for cancer. Breast J 12: 331-337, 2006.

24. Schnitt SJ, Connolly JL, Khettry U, Mazoujian G, Brenner M, Silver B, Recht A, Beadle G and Harris JR: Pathologic findings on re-excision of the primary site in breast cancer patient considered for treatment by primary radiation therapy. Cancer 59: 675-681, 1987.

25. Solin LJ, Fourquet A, Vicini FA, Haffty B, Taylor M, McCormick B, McNeese M, Pierce LJ, Landmann C, Olivotto IA, et al: Mammographically detected ductal carcinoma in situ of the breast treated with breast-conserving surgery and definitive breast irradiation: Long-term outcome and prognostic significance of patient age and margin status. Int J Radiat Oncol Biol Phys 50: 991-1002, 2001.

26. Cilotti A, Bagnolesi P, Moretti M, Gibilisco G, Bulleri A, Macaluso AM and Bartolozzi C: Comparison of the diagnostic performance of high-frequency US as a first- or second-line diagnostic tool in non-palpable lesions of the breast. Eur Radiol 7: 1240-1244, 1997.

27. Jackson VP: The current role of ultrasonography in breast imaging. Radiol Clin North Am 33: 1161-1170, 1995.

28. Wasif N, Garreau J, Terando A, Kirsch D, Mund DF and Giuliano AE: MRI versus ultrasonography and mammography for preoperative assessment of breast cancer. Am Surg 75: 970-975, 2009.

29. Ernster VL and Barclay J: Increases in ductal carcinoma in situ (DCIS) of the breast in relation to mammography: A dilemma. J Natl Cancer Inst Monogr 22: 151-156, 1997.

30. Miller AR, Brandao G, Prihoda TJ, Hill C, Cruz AB Jr and Yeh IT: Positive margins following surgical resection of breast carcinoma: Analysis of pathologic correlates. J Surg Oncol 86: $134-140,2004$. 